\title{
Filterless All-Normal Dispersion Fiber Laser
}

\author{
K. Özgören ${ }^{1}$, F. Ö. Ilday ${ }^{2}$ \\ ${ }^{1}$ Material Science and Nanotechnology Graduate Program, Bilkent University, Ankara 06800, Turkey \\ ${ }^{2}$ Departent of Physics, Bilkent University, Ankara 06800, Turkey
}

\begin{abstract}
We demonstrate mode-locked operation of an allnormal dispersion $\mathrm{Yb}$-fiber oscillator without the use of bulk bandpass filter. A section of PM-fiber incorporated into the cavity acts as a filter, paving the way towards an all-fiber oscillator based on off-the-shelf components.
\end{abstract}

All-normal-dispersion (ANDi) fiber lasers [1] are attractive laser designs due to their simpler cavity configuration in comparison to other types of Yb-doped fiber lasers which require the use of diffraction gratings or special fibers with anomalous dispersion at $1 \mu \mathrm{m}$. The mode-locked operation of these lasers requires a bandpass filter, typically an interference or a birefringent filter. However, filters are bulk components and not compatible with all-fiber oscillator designs. An allfiber design requires specialty components. In this paper, we demonstrate that a proper length PM-fiber section inserted into the cavity acts as a filter.

Fig 1. shows an ANDi type Yb-fiber laser oscillator incorporating an collimator with PM-fiber following the polarizing beam splitter (PBS), instead of the usual bandpass filter. Here, the birefringent filter acts as a fiber-based birefringence filter. After the PBS, the beam is linearly polarized. The PM fiber is aligned such that the slow and fast axes of the fiber is at $45^{\circ}$ angle with respect to the polarization of the beam.

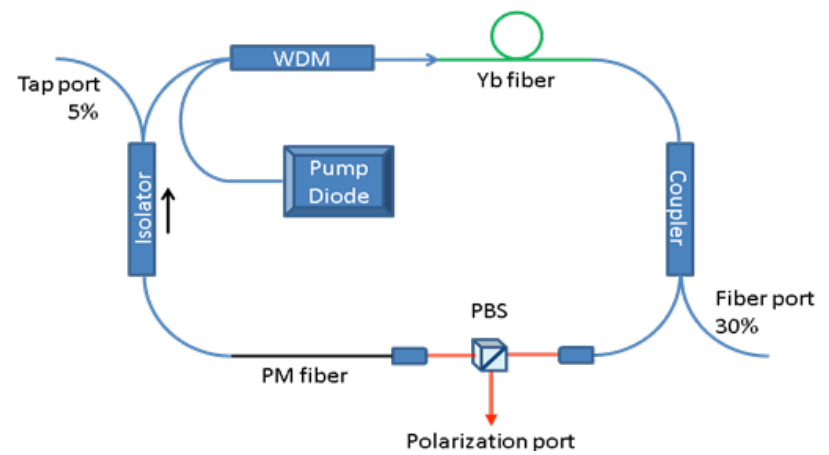

Fig. 1. Schematic of the filterless ANDi fiber laser, WDM: Wavelength division multiplexer, PBS: Polarizing beam spliter.

Beat length of the PM-fiber is specified as $\sim 2.7 \mathrm{~mm}$ at 980 $\mathrm{nm}$ wavelength which corresponds to a birefringence of $\Delta n / n=3.6 \times 10^{-4}$. Upon propagation through this fiber, there is an accumulated phase difference between the slow and fast axes given by $\Delta \phi=k L \Delta n / n$, where $k=2 \pi / \lambda$ is the wavenumber and $L$ is the length of the PM fiber. Following the PM fiber, the superposition of ligth in the two axes copropoagate throughout the cavity. The same PBS that functions as both the first and the second polarizer, constituting a simple Lyot filter [2], where the PM fiber section fulfills the role of the birefringent plate placed at an angle of $45^{\circ}$ in a conventional Lyot filter. The transmission will be maximum at the PBS if $\Delta \phi$ is a multiple of $2 \pi$. Two successive wavelengths ( $\lambda$ and $\left.\lambda^{\prime}\right)$ satisfying this condition are:

$$
\begin{aligned}
& 2 \pi m=\frac{2 \pi}{\lambda} L \frac{\Delta n}{n} \\
& 2 \pi(m+1)=\frac{2 \pi}{\lambda^{\prime}} \iota \frac{\Delta n}{n}
\end{aligned}
$$

where $m$ is an integer. Since $m \gg 1$, the effective filtering bandwidth of the PM-fiber section is given by the following expression:

$$
\Delta \lambda=L \frac{\Delta n}{n}\left[\frac{1}{m}-\frac{1}{m+1}\right]=L \frac{\Delta n}{n} \frac{1}{m^{2}}=\frac{\lambda^{2}}{L} \frac{n}{\Delta n}
$$

The pump diode operates at $500 \mathrm{~mA}$ giving an optical output power of $300 \mathrm{~mW} .30 \mathrm{~cm}$ of highly doped Yb-fiber is used as the gain medium. Mode-locked output power of the laser for both cases are similar; $40 \mathrm{~mW}$ from the fiber port and $70 \mathrm{~mW}$ from the polarization port. The repetition rate of the oscillator is $42 \mathrm{MHz}$, which corresponds to $1 \mathrm{~nJ}(3 \mathrm{~nJ})$ pulse energy from the fiber port (intracavity). Fig. 2 shows the spectra of the laser from different ports.

For the experiments, PM fiber lengths are chosen to correspond to 6.5 and $10 \mathrm{~nm}$ effective bandwidths. In both cases, mode-locking of the laser is achieved easily, though not as easily as a conventional ANDi laser with a bulk filter. Longterm operation is quite robust. Output power, optical spectrum, autocorrelation (maximum range of $15 \mathrm{ps)}$ ) and $\mathrm{RF}$ spectrum (12 GHz bandwidth) measurements are used to characterize the modelocked operation.

Figure 2(a) shows measured spectra for continous-wave (cw) operation for $6.5 \mathrm{~nm}$ and $10 \mathrm{~nm}$ effective bandwidths which shows that lasing occurs at specific wavelengths. The distances between the peaks reveal the $\Delta \lambda$ value. At a central wavelength of $1030 \mathrm{~nm}$, we calculate the required PM-fiber lengths for $6.5 \mathrm{~nm}$ and $10 \mathrm{~nm}$ effective bandwidths to be $45 \mathrm{~cm}$ and $30 \mathrm{~cm}$, respectively. These are in reasonably good agreement with the experimental values of $37 \mathrm{~cm}$ and $24 \mathrm{~cm}$, respectively. We attribute the difference to the large uncertainty of the beat length specification for the PM fiber. According to equation (3), the product of the fiber length and 
filter bandwidth should be constant. Indeed, measured bandwidth and PM fiber length products agree within $0.2 \%$.
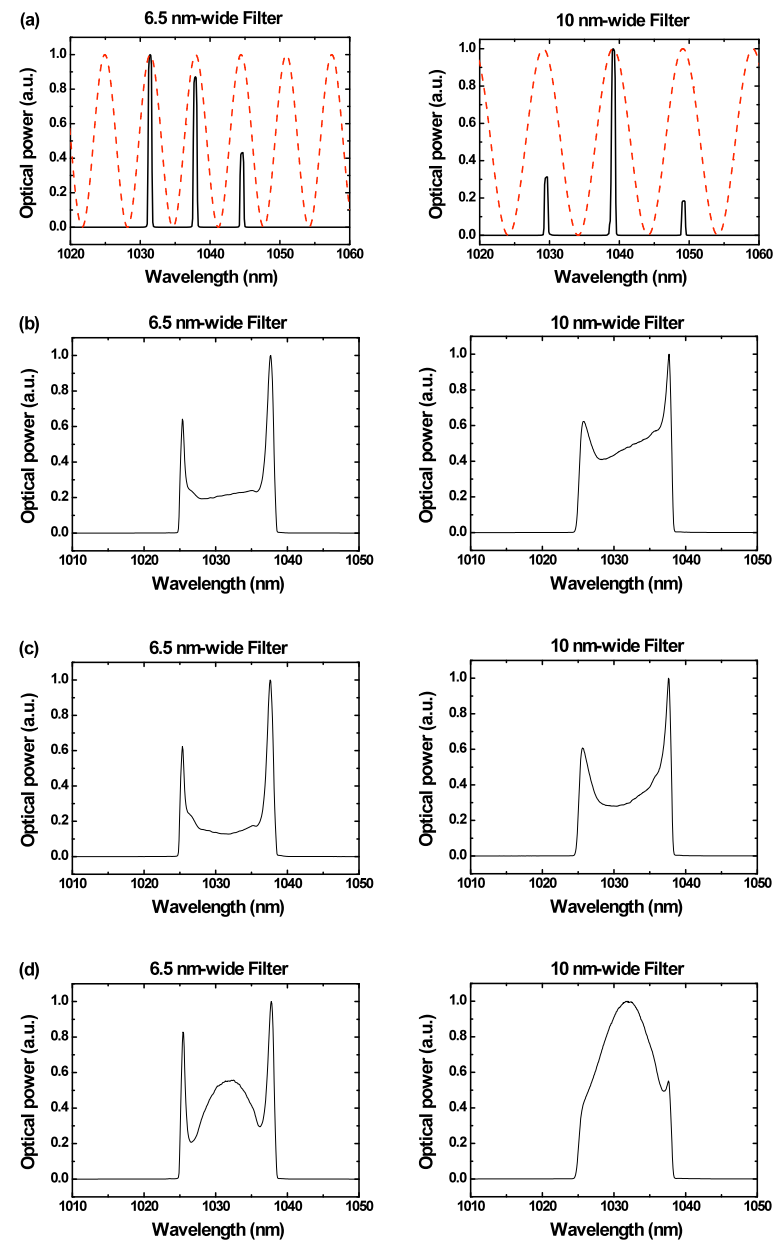

Fig. 2. Various spectra from the laser, left column shows operation with 6.5 $\mathrm{nm}$ bandwidth and right column shows operation with $10 \mathrm{~nm}$ effective bandwidth: (a) depicts spectra for $\mathrm{cw}$ operation (black lines) and sinusoidal curve-fits with a period equal to the bandwidth (red lines); (b) depicts modelocked spectra recorded from the fiber port; (c) depicts mode-locked spectra recorded from the polarization port; (d) depicts mode-locked spectra recorded from the tap port
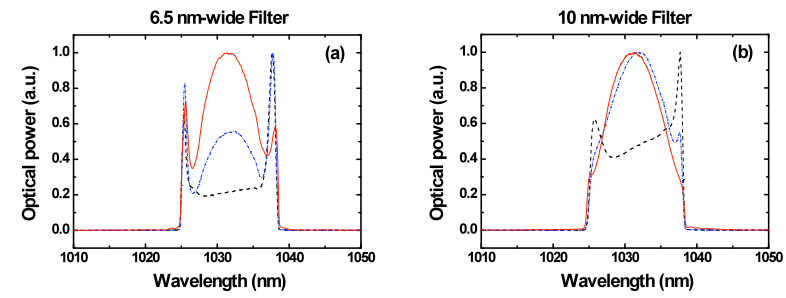

Fig. 3. Spectra recorded from the fiber port (black lines), tap port (blue lines) along with the frequency-dependent transmission for effective filter (red lines) for (a) $6.5 \mathrm{~nm}$ and (b) $10 \mathrm{~nm}$ effective bandwidths.

Pulse spectra and power measurements recorded from the polarization, fiber and tap ports for mode-locked operation allow us to reconstruct the spectral evolution in detail (Fig. 2 (b-d). It emerges that the only significant filtering occuring within the cavity takes place at the PBS. This is followed by reshaping and broadening of the spectrum during propagation through the SMF and the gain fiber. Fig. 3 shows the spectral transfer function at the PBS, which is calculated by dividing the spectrum of the tap port to fiber port following proper normalization of the amplitudes based on measurement powers at these ports. Comparison with the spectrum of the pulse rejected by the PBS and power values constitutes a sanity check for the calculation. We conclude that the PBS in this laser assumes both the roles of conversion of polarization rotation to amplitude modulation for the NPE scheme and bandpass filtering as in a Lyot filter. Although the details of this mechanism and the effect of the group velocity mismatch in the PM fiber are not well understood, the dual role of the PBS here is compatible with the fact that a saturable absorber acting on a chirped pulse also acts as a bandpass filter.

Fig. 4 shows the autocorrelation trace and RF spectrum measured at the fiber port. Compression with a grating pair gives minimum pulse durations of $240 \mathrm{fs}$ and $250 \mathrm{fs}$ for the effective bandwidths of $6.5 \mathrm{~nm}$ and $10 \mathrm{~nm}$, respectively. Highbandwidth RF spectrum of the laser shows no sign of multipulsing and close-in of the RF spectrum (Fig. 4(c-d)) shows low-noise operation.
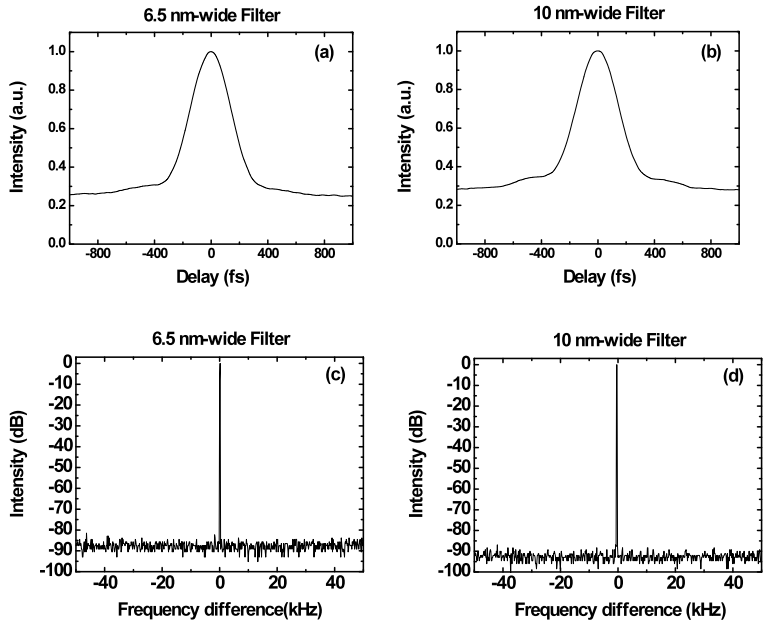

Fig. 4. Measured autocorrelation of dechirped pulses from the fiber port for (a) $6.5 \mathrm{~nm}$ and (b) $10 \mathrm{~nm}$ effective bandwidth. Measured RF spectra of the laser for (c) $6.5 \mathrm{~nm}$ and (d) $10 \mathrm{~nm}$ effective bandwidth. The horizontal axes depict frequency offset from the first harmonic.

In conclusion, we demonstrated mode-locked operation of a filterless all-normal dispersion fiber laser, where a section of PM fiber provides effective birefringence filtering. This approach has two advantages: (i) this is effectively a low-cost filter with continously adjustable bandwidth (through the fiber length), (ii) the fiber-based filtering action allows all-fiber cavity designs since fiber-coupled polarizers are available.

\section{References}

[1] B. Lyot, "Un monochromateur a grand champ utilisant les interferences en lumiere polarisee," C. R. Acad. Sci., 197, pp. 1593-1595 (1933).

[2] A. Chong, J. Buckley, W. Renninger, and F. Wise, "All-normal-dispersion femtosecond fiber laser," Opt. Express $\backslash$ textbf $\{14\}, 10095$ (2006). 